$\begin{array}{cl}\begin{array}{cl}\text { Revue } \\ \text { de Ihistoire }\end{array} & \text { Revue de l'histoire des religions } \\ \text { des religions } & 4 \mid 2010 \\ & \text { Qu'est-ce qu'un « paysage religieux »? }\end{array}$

Le paysage religieux de Sparte sous le HautEmpire

The Religious Landscape of Sparta under the High Empire

\title{
Olivier Gengler
}

\section{(2) OpenEdition \\ Journals}

Édition électronique

URL : http://journals.openedition.org/rhr/7678

DOI : $10.4000 /$ rhr.7678

ISSN : 2105-2573

Éditeur

Armand Colin

Édition imprimée

Date de publication : 1 décembre 2010

Pagination : 609-637

ISBN : 978-2200-92658-8

ISSN : 0035-1423

Référence électronique

Olivier Gengler, «Le paysage religieux de Sparte sous le Haut-Empire », Revue de l'histoire des religions

[En ligne], 4 | 2010, mis en ligne le 01 décembre 2013, consulté le 20 avril 2019. URL : http://

journals.openedition.org/rhr/7678; DOI : 10.4000/rhr.7678 


\section{Le paysage religieux de Sparte sous le Haut-Empire}

Cette étude présente les premiers résultats d'une enquête portant sur le paysage religieux de Sparte aux trois premiers siècles de notre ère, essentiellement à travers l'analyse de la place occupée par les lieux de culte et les pratiques rituelles dans l'organisation et dans les représentations symboliques du territoire de la cité. Après une présentation du contexte politique et économique mettant l'accent sur les problèmes posés par les sources, nous nous attacherons à la distribution des lieux de culte dans le territoire, aux parcours qui unissent certains d'entre eux ensemble et à la ville, et enfin à la présence des femmes de Sparte dans le sanctuaire de Déméter Eleusinia.

\section{The Religious Landscape of Sparta under the High Empire}

This paper gives the first results of a research project regarding the religious landscape of Sparta during the first three centuries AD, mainly through the analysis of the role of places of worship and ritual practices in the organization of the city and in symbolic representations of its territory. After a presentation of the political and economic context, stressing the problems of interpreting the available sources, I will study the distribution of the places of worship in the territory, the routes that link some of them together and to the town, and finally the presence of Spartan women in the sanctuary of Demeter Eleusinia. 
Toute société humaine construit l'espace dans lequel elle s'inscrit à travers les pratiques qu'elle y déploie, les aménagements qu'elle y apporte et les représentations symboliques dont elle l'investit. En retour, l'espace modèle la société qui y évolue. L'espace comme construction sociale n'est donc ni immuable, ni univoque. Les valeurs qui lui sont associées sont susceptibles d'évoluer avec le temps, de même que des valeurs différentes, émanant de différents groupes sociaux, peuvent y être projetées simultanément ${ }^{1}$.

Nous voudrions, dans cette étude, présenter les premiers résultats d'une enquête portant sur le paysage religieux de Sparte sous le Haut-Empire, soit dans les trois premiers siècles de notre ère. Dans la mesure de ce que nous permet la documentation, il s'agira de voir comment s'organise et comment était perçue la présence du religieux dans le territoire d'une des principales cités de la Grèce classique, dans le cadre politique et culturel de l'empire romain². Ce sera l'occasion de revenir sur le modèle développé par Susan E. Alcock il y a un peu plus de quinze ans pour rendre compte de l'évolution du «paysage sacré » en Grèce à l'époque romaine 3 .

L'importance des pratiques religieuses dans la société civique grecque légitime pleinement selon nous d'en privilégier l'étude, malgré ce que le découpage d'un "paysage religieux » peut avoir d'artificiel. En effet, même si, à fin d'analyse, il est utile de notre point de vue moderne d'isoler, dans l'espace construit par une société déterminée, ce qui touche à la religion et au sacré, cette dimension n'en reste pas moins indissociable d'un cadre politique

1. Pour une approche générale, voir Anne Cadoret, «Espace », in : Pierre Bonte, Michel Izard (éd.), Dictionnaire de l'ethnologie et de l'anthropologie, Paris, $2000^{3}$, p. 235-236.

2. Cette étude est une version remaniée de notre communication au colloque «Qu'est-ce qu'un paysage religieux? », présentée à l'invitation de F. de Polignac et de J. Scheid, que nous tenons à remercier ici. Elle s'appuie sur le matériel réuni dans le cadre de notre collaboration au programme ANR «CIRCE » et s'inscrit dans un projet de recherche sur la cité de Sparte à l'époque impériale, financé par la Fondation Gerda Henkel.

3. Susan E. Alcock, Graecia Capta. The Landscapes of Roman Greece, Cambridge, 1992, p. 200-214. Pour une évaluation critique du modèle développé par Susan E. Alcock dans son livre, voir le bilan convaincant et très nuancé de Denis Rousset, « La cité et son territoire dans la province d'Achaïe et la notion de "Grèce Romaine" ", Annales. Histoire, Sciences Sociales 59-2 (2004), p. 363-383. 
ou économique, qu'il nous faut donc retracer à grands traits pour la région et l'époque qui vont nous occuper ici.

\section{LA LACONIE D'ÉPOQUE IMPÉRIALE}

Depuis sa victoire sur la Ligue Achéenne, marquée par la destruction complète de Corinthe en 146, Rome exerce sa tutelle sur la Grèce. Cet événement politique a longtemps été perçu également comme une rupture dans l'histoire économique ou culturelle de ce qui, en 27 avant J.-C., devint la province d'Achaïe ${ }^{4}$ Pourtant, il apparaît que la vie des cités grecques s'est poursuivie sous une forme qui, si elle n'est pas demeurée inchangée, ne doit en tout cas pas être vue comme un déclin. Il faut en effet se départir de l'image transmise par les textes qui dépeignent parfois une Grèce dévastée.

Aux yeux de Strabon, qui rédige une description géographique du monde méditerranéen au début de l'ère chrétienne, la vieille Grèce est malade : les cités ont disparu, les campagnes sont vides. À la fin de sa description de la Messénie, avant d'en venir à la Laconie, le géographe semble contrit d'avoir à tant parler de ces régions :

« Cependant, emporté par l'abondance des données, nous en venons à parler outre mesure d'un pays en majeure partie abandonné. En fait, la Laconie aussi est dépeuplée, si on en juge par la comparaison avec la population florissante qu'elle eut jadis. En dehors de Sparte, il n'y a que des petites villes, au nombre de trente environ. Or, jadis, on qualifiait [la Laconie] d'hécatompole, à ce qu'on dit, et pour cette raison on offrait chez eux le sacrifice des Hécatombes chaque année 5 . »

Il est certain que la région devait alors encore porter les stigmates de plusieurs décennies de guerres et de troubles : guerres de Mithridate, incursions de pirates, guerres civiles ${ }^{6}$. Sans avoir véritable-

4. Voir par exemple Athanasios D. Rizakis, «Les cités péloponnésiennes entre l'époque hellénistique et l'Empire : le paysage économique et social», in : Regula Frei-Stolba, Kristine Gex (éd.), Recherches récentes sur le monde hellénistique, Berne, 2001 [ECHO 1], p. 75-96.

5. Strabon, VIII, 4, 11 (C 362). Cf. Eustathe, Commentaire à Denys le Périégète, 419, qui ne semble pas ici dépendre de Strabon.

6. Pour le cadre général, voir Jean-Marie Bertrand, «Rome et la Méditerranée orientale au $\mathrm{I}^{\text {er }}$ siècle avant J.-C. », in : Claude Nicolet (éd.), Rome et la conquête du monde méditerranéen, 2 : Genèse d'un empire, Paris, $1989^{2}$ [Nouvelle Clio, 8 bis], p. 789-845, spécialement p. 834-840 pour les aspects économiques. Pour Sparte et la Laconie, voir : Paul Cartledge, Antony J.S. Spawforth, 
ment constitué l'enjeu de ces conflits qui marquent le dernier siècle de la république romaine, le Péloponnèse en fut parfois le théâtre et souvent la victime. Le statut de cité libre et immune dont bénéficiait Sparte ne la mettait pas à l'abri des réquisitions, considérées comme des devoirs d'amitié envers Rome ${ }^{7}$.

L'impact des guerres du i ${ }^{\text {er }}$ siècle avant J.-C. sur le Péloponnèse en général et sur la Laconie et Sparte en particulier peut donc justifier sans doute, pour une part, le jugement de Strabon. Pour autant, celui-ci semble exagéré, en raison tout d'abord d'une erreur d'appréciation. Quelle réalité en effet l'image d'une Laconie «aux cent villes » recouvre-t-elle? Stéphane de Byzance, dans ses Ethnica, sorte de dictionnaire de géographie composé au $\mathrm{VI}^{\mathrm{e}}$ siècle après J.-C., compte « au nombre des cent » quelques cités qu'il présente comme laconiennes mais aussi des cités appartenant à la Messénie hellénistique et romaine, jadis soumise à Sparte ${ }^{8}$. La Laconie « aux cent villes » est donc une unité politique, le territoire dominé par Sparte au faîte de sa puissance, lorsque elle exerçait son pouvoir sur l'équivalent des deux cinquièmes du Péloponnèse selon l'expression de Thucydide ${ }^{9}$, et non la région géographique, bien plus étroite, à laquelle se réfère ici Strabon ${ }^{10}$. Or le territoire de la cité de Sparte a beaucoup évolué depuis la fin de l'époque classique ${ }^{11}$. Après avoir

Hellenistic and Roman Sparta. A Tale of two Cities, Londres - New York, 20022, p. 94-97 avec Raoul Baladié, Le Péloponnèse de Strabon. Étude de géographie historique, Paris, 1980, p. 301-311.

7. Strabon, VIII, 5,5 (C 365) avec Silvio Accame, Il Dominio romano in Grecia dalla guerra acaica ad Augusto, Rome, 1946 [Studi pubblicati dal R. Istituto Italiano per la Storia Antica, 4], p. 129-130.

8. Respectivement Aphrodisias (Stéphane de Byzance, s.v.) et Aithaia (Stéphane de Byzance, s.v.; $c f$. Thucydide, I, 101, 2) ou Aulon (Stéphane de Byzance, s.v.; cf. Pausanias, IV, 36, 7). Cf. Graham Shipley, " "The Other Lakedaimonians" : The Dependent Perioikic Poleis of Laconia and Messenia », in : Mogens Herman Hansen (éd.), The polis as an urban centre and as a political community, Copenhague, 1997 [Det Kongelige Danske Videnskabernes Selskab, Historisk-filosofiske Meddelelser, 75/Acts of the Copenhagen Polis Centre, 4], p. 225.

9. Thucydide, I, 10, 2 avec le commentaire de Arnold W. Gomme, $A$ Historical Commentary on Thucydides, t. I, Oxford, 1945, p. 112 sur le sens à donner à « Laconie » dans ce contexte. Strabon, VIII, 5, 3 (C 364) emploie également Laconie en ce sens quand il dit que Messène en faisait partie.

10. Essentiellement comprise entre le Taygète et le Parnon : Strabon, VIII, 5, 1 (C 362-363) et 6, 1 (C 368).

11. Voir Graham Shipley, "The extent of Spartan territory in the Late Classical and Hellenistic periods ", Annual of the British School at Athens [dorénavant $A B S A] 95$ (2000), p. 367-390. 
perdu une grande partie de la Messénie après Leuctres, en 371 avant J.-C., après en avoir perdu le reste par décision de Philippe II de Macédoine après Chéronée en 338, ainsi sans doute qu'une partie des cités du versant oriental du Parnon, Sparte avait en effet dû renoncer à sa domination sur toutes les cités de la côte après la défaite de Nabis devant Flamininus. À l'époque de Strabon, Sparte domine encore la plaine de l'Eurotas, entre le Taygète et le Parnon, ainsi que quelques cités sur le versant occidental du Taygète : Thouria, Pharai et Cardamylé, de même que l'île de Cythère. Ces cités ont été attribuées à Sparte par Auguste en récompense de l'engagement lacédémonien à ses côtés lors du combat devant Actium $^{12}$ et en guise de compensation après que les autres cités du littoral se soient vu confirmer leur indépendance au sein de la ligue des Laconiens libres.

Le jugement de Strabon révèle également une conception exagérée de l'antique prospérité de la région. Si même la représentation d'une Laconie « aux cent villes » n'est pas le fruit d'une reconstruction érudite, forgée précisément d'après le nom de la fête des Hécatombes qu'évoque Strabon ${ }^{13}$, il faut certainement y voir une hyperbole soulignant la puissance de Sparte à l'époque classique : son territoire était alors assez vaste, comptait assez de villes pour qu'on s'imaginât qu'il en contenait cent. Plus généralement, l'idée du déclin de la Grèce, qui constitue un véritable topos chez les auteurs d'époque impériale et dont on trouve un écho notamment dans le discours de Néron à l'Isthme en 67 après J.-C. ${ }^{14}$, s'explique de même comme un reflet, inverse, de l'image idéalisée du passé de la Grèce, image qui est elle-même une construction littéraire ${ }^{15}$.

12. Pausanias, IV, 31, 1-2; cf. VIII, 8, 12. Voir Karim Arafat, Pausanias' Greece. Ancient artists and Roman rulers, Cambridge, 1996, p. 132-134.

13. Fête qui a pu être effectivement célébrée par les Lacédémoniens dont le calendrier comportait un mois Hecatombeus : Hésychios, Epsilon 1272 Latte.

14. Sylloge Inscriptionum Graecarum, $3^{\text {e }}$ éd., 814, 1. 16-18. Voir Jakob A. O. Larsen, « Roman Greece», in : Frank Teeney (éd.), An Economic Survey of Ancient Rome, IV, Baltimore, 1938, p. 259-498.

15. Pour la critique des documents littéraires nous informant de la situation économique de la Grèce romaine, voir les remarques générales de J.A.O. Larsen, « Roman Greece », art. cité, p. 465, et de Maurice Sartre, L'Orient romain, Paris, 1991, p. 212. Le jugement de Strabon s'inscrit dans tout un système de représentations associées à l'idée de peuplement : voir Luigi Gallo, «Popolosità e scarsità di popolazione. Contributo allo studio di un topos », Annali della Scuola Normale Superiore di Pisa, III" série, 10 (1980), p. 1233-1270. 
Il est avéré que des établissements humains ont disparu en Laconie, sans l'ampleur toutefois que laisserait supposer le témoignage de Strabon et, surtout, sans qu'il soit possible d'y voir une conséquence directe de la conquête romaine ou de l'instabilité politique de la fin de la République. De toutes les cités de Laconie que Pausanias, dans sa Description de la Grèce rédigée sous le règne de Marc Aurèle, signale en ruine, bien peu ont disparu de son temps, quand, à l'instar de Pharis et Bryséai sorties tout droit d'Homère ${ }^{16}$, elles ne sont pas de simples souvenirs littéraires. Le paysage politique a donc évolué, les frontières de la cité de Sparte ont été redessinées et, en leur sein, certains établissements humains ont sans doute périclité, mais cela ne fait pas de la région un désert, irrémédiablement dépeuplé.

Une prospection archéologique systématique dans la plaine et les collines qui s'étendent à l'est de Sparte, sur la rive gauche de l'Eurotas, entreprise sur une surface d'environ $70 \mathrm{~km}^{2}$, a montré une réduction significative des sites où l'on peut reconnaître une activité humaine d'habitat ou de production à l'époque romaine. Ainsi, de soixante-quinze sites à l'époque hellénistique - considérée ici comme les trois derniers siècles avant J.-C. -, on passe à cinquante et un sites pour les six premiers siècles après J.-C., soit à une réduction d'un tiers de sites sur une période archéologique deux fois plus longue. En outre, les sites apparaissent de moindre importance et ne dépassent jamais, au mieux, la taille d'un hameau, là où on pouvait précédemment trouver des villages. Ces résultats sont toutefois à considérer avec prudence ${ }^{17}$. La chronologie, notamment, soulève plusieurs réserves, dans la mesure où la périodisation retenue semble relativement arbitraire et où la mauvaise connaissance de la céramique laconienne commune post-classique demeure de toute manière insuffisante ${ }^{18}$. Par ailleurs, les données manquent

16. Iliade, II, 582-583.

17. Pour une discussion d'ensemble, nous renvoyons à l'analyse de D. Rousset, «La cité et son territoire dans la province d'Achaïe et la notion de "Grèce Romaine" ", art. cité, spécialement aux p. 365-373.

18. Voir les remarques de Heleen Visscher et Jo Lawson, in: William Cavanagh, Richard Catling, Joost Crouwel., Continuity and Change in a Greek Rural Landscape : the Laconia Survey, II : Archaeological Data, Londres, 1996 [British School at Athens, Supplementary vol. 27], p. 91, 111. Depuis, l'étude systématique du matériel mis au jour dans les fouilles du théâtre et de la stoa romaine a permis de combler partiellement cette lacune : Clare Pickersgill, Paul Roberts, « New Light on Roman Sparta : Roman Pottery from the Sparta Theatre and Stoa », ABSA 98 (2003), p. 549-597. 
pour évaluer la durée de vie de ces différents sites au sein même des périodes ainsi distinguées et pour retracer donc de manière fine l'évolution du paysage. Les variations, d'une période à l'autre, dans le profil des sites et dans la densité d'occupation apportent cependant quelques indices intéressants. Le matériel céramique des sites occupés durant les six premiers siècles de notre ère se distingue de celui des époques précédentes par une nette prédominance des récipients de stockage et une diminution des formes associées à la vie domestique, ce qui engagerait à voir dans les sites explorés non des habitats, mais des structures liées à l'exploitation agricole ${ }^{19}$. Par ailleurs, la diminution du nombre de sites est plus marquée à proximité de la ville, où se concentrerait donc désormais l'habitat.

Il est donc probable que l'on assiste à un redéploiement de la structure de l'habitat et, peut-être, à une modification dans l'exploitation des terres, avec un développement de l'élevage ${ }^{20}$. En tout cas, la baisse apparente de la densité d'occupation du territoire semble aller de pair avec le développement de la ville de Sparte - du moins tel qu'il est apparu lors des nombreuses fouilles d'urgence exécutées ces trente dernières années. À partir de la fin du $\mathrm{I}^{\mathrm{er}}$ siècle avant J.-C., la ville semble se réorganiser suivant un plan orthogonal à l'intérieur des murailles hellénistiques, tandis que deux importantes nécropoles se développent en périphérie, au nord et au sud-ouest ${ }^{21}$.

19. Graham Shipley, in: William Cavanagh, Richard Catling, Joost Crouwel., Continuity and Change in a Greek Rural Landscape: the Laconia Survey, I : Methodology and Interpretation, Londres, 2002 [British School at Athens, Supplementary vol. 26], p. 334. Les données réunies par le Laconia Rural Sites Project n'ont pas conduit à une modification de ces conclusions : William Cavanagh, Christopher Mee, Peter James, The Laconia Rural Sites Project, Londres, 2005 [British School at Athens, Supplementary vol. 36], p. 299-300 et p. 315 .

20. L'élevage semble avoir effectivement constitué un pôle économique important en Laconie: Christophe Chandezon, «Les spécificités pastorales du Péloponnèse à l'époque hellénistique et sous le Haut-Empire ", in Catherine Grandjean (éd.), Le Péloponnèse d'Épaminondas à Hadrien, Bordeaux, 2008, p. 129-144. Cependant, il est moins certain qu'il se soit développé dans les proportions indiquées et selon la chronologie inférée des résultats de la prospection archéologique. Voir les réserves de D. Rousset, «La cité et son territoire dans la province d'Achaïe et la notion de "Grèce Romaine" ", art. cité, p. 371-373.

21. $C f$. Stella Raftopoulou, "New finds from Sparta», in William G. Cavanagh, Susan E.C. Walker (éd.), Sparta in Laconia. Proceedings of the 19th British Museum Classical Colloquium, Londres, 1998 [British School at Athens Studies, 4], p. 136-137 (une faute de mise en page a malheureusement fait disparaître une partie du texte); Heleni Kourinou, Sparti. Symbôli sti mnimeiaki 
La zone d'habitat s'étend et la richesse des maisons s'accroît. Les fouilles ont mis au jour un grand nombre de demeures très riches, avec des mosaïques de qualité - produites aux III ${ }^{\mathrm{e}}$ et $\mathrm{IV}^{\mathrm{e}}$ siècle par un atelier local -, et des bains ${ }^{22}$.

\section{LES CULTES DU TERRITOIRE À L'ÉPOQUE IMPÉRIALE}

Comment, dans ce cadre, a évolué le paysage religieux? Nous disposons, pour esquisser une réponse, de données archéologiques, de textes épigraphiques et, surtout, d'un témoignage littéraire : la Description de la Grèce de Pausanias. D'emblée, il faut préciser que, malgré sa richesse, ce texte ne nous donne qu'une information partielle. Il est en effet sélectif, comme l'auteur le rappelle luimême à plusieurs reprises ${ }^{23}$. En outre, une de ses caractéristiques est de mêler l'évocation de choses vues et de choses sues : l'auteur tente de retrouver dans les cités de la Grèce la trace de leur passé et agrège dans son texte traditions anciennes et évocation du présent, sans qu'il soit toujours possible de les démêler ni de les réduire à tel ou tel type de discours, narratif ou descriptif. La curiosité de Pausanias est guidée par l'intérêt qu'il porte au passé - intérêt qui est, il faut le souligner, en totale adéquation avec l'esprit de son temps. Il en résulte une tendance à préférer ce qui est ancien ou, plutôt, ce qui passait pour tel. Il s'ensuit également un certain écrasement des perspectives qui donne à l'assemblage de faits, de traditions, de monuments de différentes époques une unité qui, en dehors du discours érudit de Pausanias - et peut-être de certains de ses contemporains - n'a pas nécessairement d'existence.

topographia tis, Athènes, 2000, p. 215-219. Le dégagement du cimetière sud-ouest se poursuit : cf. Bulletin de correspondance hellénique 124 (2000), p. 815; Bulletin de correspondance hellénique 125 (2001), p. 841.

22. Outre les références données par Paul Cartledge et Antony J.S. Spawforth, Hellenistic and Roman Sparta, Appendice I, $\mathrm{n}^{\text {os }}$ 51-64, 73-74, p. 222-225, voir S. Raftopoulou, « New finds from Sparta », art. cité, p. 127-133. Sur les mosaïques, voir plus spécialement Anastasia Panagiotopoulou, "Roman Mosaics from Sparta », in : W.G. Cavanagh, S.E.C. Walker (éd.), Sparta in Laconia, op. cit., p. 112-118; Id., «Un atelier de mosaïques romaines à Sparte », in : Daniel Paunier et Christophe Schmidt (éd.), La mosaïque gréco-romaine, VIII, Lausanne, 2001, t. 1, p. $238-248$.

23. Cf. Pausanias, I, 39, 3 et III, $11,1$. 


\section{Les cultes du territoire}

On connaît pour la période romaine une quarantaine de lieux de culte sur le territoire de la cité de Sparte ${ }^{24}$. Trente et un sont signalés par Pausanias (sanctuaires, temples, statues divines ou héroïques), parmi lesquels cinq ont été également repérés sur le terrain ${ }^{25}$. Deux sanctuaires sont connus par d'autres sources littéraires, sans que l'on puisse être totalement certain qu'ils aient été encore en activité à l'époque impériale - le fait que Pausanias ne les mentionne pas n'étant pas un critère déterminant de ce point de vue ${ }^{26}$. Deux autres sanctuaires repérés archéologiquement, mais restés anonymes, pourraient avoir été en activité à l'époque impériale, et correspondre par ailleurs à l'un ou l'autre des lieux de culte signalés par Pausanias ${ }^{27}$. Enfin, un sanctuaire de Zeus Messapeus, différent de celui qui est connu par Pausanias, semble renaître aux III $^{\mathrm{e}}$-IV ${ }^{\mathrm{e}}$ siècle après J.-C. ${ }^{28}$, de même qu'un sanctuaire de divinité féminine, sans doute Artémis, localisé à proximité d'Aigai ${ }^{29}$. Sur les cinq sanctuaires connus par Pausanias et l'archéologie, deux présentent une activité assurée à l'époque impériale : l'Éleusinion et l'Amyclaion, et deux une activité probable: le sanctuaire de Zeus Messapeus et le sanctuaire d'Agamemnon et d'Alexandra. Un seul, le Ménélaion, n'a livré aucune trace d'activité cultuelle pour cette période.

Que représentent ces chiffres? Pour Susan E. Alcock, même si Pausanias signale un certain nombre de lieux de cultes à l'état d'abandon - dont la ruine, du moins pour une partie d'entre eux, serait selon elle imputable aux troubles du $\mathrm{I}^{\mathrm{er}}$ siècle avant J.-C., à la crise économique et donc à la désertification des campagnes -, c'est surtout le maintien de la majorité des lieux de cultes ruraux qu'il faudrait expliquer. Selon son estimation en effet, pour un

24. Voir le catalogue ci-dessous. Pour les questions de topographie, voir notre commentaire au livre III de Pausanias, à paraître dans la collection Budé.

25. B9, B10, B14, B17 et C29.

26. A4 et B8.

27. D31 et B6. Le premier pourrait correspondre à n'importe lequel des sanctuaires mentionnés par Pausanias en III, 20, 8-10, tous situés à proximité de Sparte, le long de la route d'Arcadie. Le second pourrait être identifié au sanctuaire d'Artémis Corythalia B8 (voir supra note 27), à celui des Charites B7, ou à l'hérôon de Lacédémon B13.

28. Sanctuaire repéré lors du Survey à Tsakona, au-dessus d'Aphyssou, au nord-est de Sparte : C25; $c f$. B14.

29. B22. 
sanctuaire en ruine, Pausanias en a vu douze qui se sont maintenus. Bien plus, leur nombre étonne en comparaison de celui des centres d'habitat connus à son époque ${ }^{30}$. Cependant, comme nous l'avons souligné d'emblée, le témoignage de Pausanias doit être correctement interprété. On ne peut notamment exclure qu'il livre une image du territoire des cités qui ne correspond déjà plus à la réalité cultuelle de son temps ${ }^{31}$. Par ailleurs, Pausanias privilégie les éléments remarquables du paysage, avec une prédilection pour ce qui touche au religieux. À l'inverse, il est probable que les prospections archéologiques de surface ne sont pas toujours en mesure de repérer ou d'identifier les lieux de culte ${ }^{32}$. Par conséquent, il est extrêmement difficile de corréler le nombre de sanctuaires, connus essentiellement par Pausanias, à la densité d'occupation d'un territoire, reconstruite uniquement à partir des découvertes archéologiques.

Trop peu de sanctuaires sont assez précisément localisés pour procéder à une véritable étude de répartition ${ }^{33}$. En outre, il n'est pas absolument certain que tous les sanctuaires connus pour l'époque impériale aient fonctionné simultanément, même ceux que mentionne Pausanias ${ }^{34}$. De plus, nous ne pouvons être assurés de connaître l'ensemble des sanctuaires actifs à l'époque. Cependant, quelques tendances se dessinent.

Parmi les sanctuaires connus, un grand nombre se trouvaient à proximité de la ville et une majorité dans un rayon de cinq kilomètres à partir de ses remparts ${ }^{35}$. Cette limite est relativement arbi-

30. S. E. Alcock, Graecia Capta, The landscapes of Roman Greece, op. cit., p. 201 cite l'exemple du Sud de l'Argolide où Pausanias connaît plus de sanctuaires que les prospections archéologiques n'ont révélé de sites ( $c f$. p. 40-41 et fig. 13).

31. L'argument est écarté un peu rapidement par Susan E. Alcock, « Minding de Gap in Hellenistic and Roman Greece », in : Susan E. Alcock, Robin Osborne, Placing the Gods. Sanctuaries and Sacred Space in Ancient Greece, Oxford, 1994, p. 258 : que Pausanias signale l'état d'abandon de certains lieux de culte ne veut pas dire qu'il l'ait fait systématiquement, ni ne permet de déterminer à partir de quel degré d'abandon ou de quel degré de vénérabilité du sanctuaire concerné, il a cru bon de le signaler.

32. Voir les remarques de Michael H. Jameson, " Mapping Greek Cults », in: Frank Kolb (éd.), Chora und Polis, Munich, 2004, p. 157, et de Susan E. Alcock elle-même, S. E. Alcock, Graecia Capta, The landscapes of Roman Greece, op. cit., p. 175.

33. Sont exactement localisés : B6, B9, B10, B14, B17, B22, C25, C29, D31.

34. Voir ci-dessus.

35. A5, B6-B8, B12, C25, C26-C30, D31-D37. On peut sans doute y ajouter encore $\mathrm{B} 13$. 
traire : c'est celle que nous permettent de fixer nos sources. Elle a cependant un certain sens, puisqu'elle délimite une zone où les sanctuaires sont accessibles en une heure au plus et demeurent facilement accessibles depuis la ville. L'Éleusinion, à environ six kilomètres et demi de la ville, appartient aussi à cette première couronne. À l'époque de Pausanias, cet espace densément occupé était séparé de la ville par une muraille. Même si ce ne fut pas toujours le cas (puisque Sparte ne fut fortifiée que progressivement à partir du III ${ }^{\mathrm{e}}$ siècle avant J.-C.), cette limite est tangible, et donc probablement signifiante, à l'époque impériale.

Les sanctuaires qui se trouvent à une plus grande distance de la ville sont pour la plupart associés à des localités : Asclépios à Pellana, Zeus et les Dioscures à Crokéai, Poseidon et Artémis à Aigiai $^{36}$. Nous ne disposons d'aucune information concernant ces sanctuaires, mais il est vraisemblable qu'ils aient été fréquentés et gérés par ces communautés locales. Il n'est pas certain que le sanctuaire d'Artémis à Sellasia ait survécu à la destruction de 222 avant J.-C., mais la région est restée habitée et un culte a pu y subsister ${ }^{37}$. Aucun de ces lieux de culte ne semble avoir eu un rayonnement tel que nous puissions supposer qu'il ait été un sanctuaire civique de Sparte. Au contraire, à Amyclées, il est vraisemblable que les cultes de Dionysos Psilax, d'Alexandra et Agamemnon et d'Apollon avaient une assise à la fois locale et civique - cette dernière étant prépondérante pour le grand sanctuaire d'Apollon ${ }^{38}$.

Quelques autres sanctuaires se trouvaient encore dans la plaine de l'Eurotas ou sur les contreforts du Taygète. Nos sources ne permettent ni de les localiser précisément - à l'exception du sanctuaire de Zeus Messapeus d'Antochori s'il a été correctement identifié ${ }^{39}$-, ni de préciser s'ils étaient ou non associés à un établissement humain. Seul le culte de Zeus/Hélios sur le Taleton a un lien assuré avec Sparte ${ }^{40}$.

Reste une série de sanctuaires situés de manière assurée aux confins du territoire. Il s'agit d'abord du sanctuaire - ou des sanc-

36. Respectivement D38, B19 et B20, B21 et B22.

37. A4; pour l'occupation de la région, voir W. Cavanagh, R. Catling, J. Crouwel., Continuity and Change in a Greek Rural Landscape : the Laconia Survey, II : Archaeological Data, op. cit., p. 321-331.

38. B10, B11 et B9.

39. B14.

40. B16. Les autres sanctuaires sont ceux de Dionysos à Bryséai (B15) et d'Artémis Déréatis, dans le Taygète (B18). 
tuaires - d'Asclépios et d'Artémis Daphnaia au lieu-dit Hypsoi, « les Hauteurs », que Pausanias situe à un peu plus de cinq kilomètres de Las, mais dans les frontières de Sparte. À cette époque, à l'autre extrémité du territoire de la cité se trouvaient encore un sanctuaire de Zeus Scotitas, une statue d'Héraclès et un sanctuaire d'Artémis Caryatis. Aucun n'est précisément localisé, mais la description de Pausanias permet du moins d'identifier la zone où ils se situaient. D'après les traditions qui lui sont associées, le sanctuaire de Caryai semble avoir été desservi et fréquenté par des Spartiates. Nous ignorons si c'était encore le cas à l'époque impériale, mais plusieurs textes, outre celui de Pausanias, gardent vivant le souvenir des rituels qu'y pratiquaient les jeunes filles de la cité. Même si, à partir des sources disponibles, on ne peut mesurer l'activité cultuelle dans ces sanctuaires à l'époque impériale, il est significatif que certains d'entre eux - qui ont pu jouer un rôle de repère physique dans le paysage -, ont également exercé une telle fonction sur le plan symbolique. C'est évidemment le cas pour le sanctuaire de Caryai, qui, selon une tradition rapportée par Pausanias, avait été le théâtre d'un exploit d'Aristomène lors de la seconde guerre de Messénie. Le chef messénien se serait emparé là de jeunes spartiates qui dansaient pour Artémis lors d'une incursion sur le territoire spartiate ${ }^{41}$. Au-delà de ses connotations initiatiques, l'épisode révèle l'importance symbolique du lieu comme ancrage de la cité de Sparte aux marges de son territoire. Le sanctuaire d'Hypsoi at-il été à son tour investi d'une telle valeur après que le territoire de la cité se soit réduit au $\mathrm{II}^{\mathrm{e}}$ siècle avant J.-C.? Une réappropriation de ce type est en tout cas attestée dans la Messénie voisine au sanctuaire d'Artémis Limnatis. En effet, la cité de Messène y restaura le culte après avoir obtenu de Rome la confirmation de ses droits sur la région où se trouvait le sanctuaire, longtemps disputé à Sparte ${ }^{42}$.

Au-delà de ses frontières, la cité de Sparte entretenait encore des liens avec des sanctuaires lui ayant appartenu. Ainsi le sanctuaire

41. IV, 16, 9-10 avec Pierre Ellinger, La légende nationale phocidienne, Paris, 1993 (BCH Suppl. 27), p. 307.

42. Sur ce sanctuaire, voir Nino Luraghi, The Ancient Messenians : Constructions of Ethnicity and Memory, Cambridge, 2008, p. 16-27 et Olivier Gengler, «Héraclès, Tyndare et Hippocoon dans la description de Sparte par Pausanias : mise en espace d'une tradition mythique », Kernos 18 (2005), p. 322-328. 
de Poseidon au cap Ténare, devenu le sanctuaire fédéral de la ligue des Lacédémoniens ${ }^{43}$. Pausanias a vu dans la ville un sanctuaire de Poseidon Tainarios ainsi qu'un monument funéraire de Tainarios qui avait donné son nom au cap ${ }^{44}$. Trois stèles, datables du $\mathrm{i}^{\mathrm{er}}$ siècle avant J.-C. et découvertes à Sparte, portent également les noms des participants à une fête liée au culte de Poseidon Tainarios ${ }^{45}$. Si le sanctuaire urbain, succursale de celui du cap Ténare, n'est pas une création hellénistique, liée à la perte de contrôle de Sparte sur la côte de Laconie, il est en tout cas certain que son rôle a pu s'en retrouver accru. Quoi qu'il en soit, le centre urbain, tel que l'a vu Pausanias, conservait de nombreuses marques du rapport de Sparte avec cette partie de son ancien territoire.

\section{De la ville au territoire}

Essentiellement grâce à Pausanias, nous pouvons également reconstruire une partie des parcours rituels qui animaient le paysage que nous venons de reconstituer. Le premier de ces parcours associe un espace de la cité, le Platanistas, à deux sanctuaires des environs immédiats de Sparte : le sanctuaire d'Achille et le Phoibaion ${ }^{46}$. Sous la surveillance officielle de magistrats, les Bidiaioi, les jeunes spartiates engagés dans la formation éphébique procédaient à un combat ritualisé les opposant les uns aux autres dans un espace délimité par un canal et entouré de platanes, appelé Platanistas. Répartis en deux groupes, les participants devaient se rendre maîtres du terrain en poussant leurs adversaires dans l'eau. Dans la nuit qui précédait ce combat, les jeunes gens sacrifiaient des chiots au dieu guerrier Enyalios dans l'enceinte du Phoibaion, un sanctuaire situé en dehors de la ville, au pied du Ménélaion qui accueillait également un culte des Dioscures ${ }^{47}$.

43. Cf. Inscriptiones Graecae, t. V, fasc 1, 1226-1227 et 1336: Laconia et Messenia, éd. par Walter Kolbe, Berlin, 1913 [dorénavant $I G$ V1], avec Nigel M. Kennell, « From Perioikoi to Poleis. The Laconian Cities in the late hellenistic period», in : Stephen Hodkinson, Anton Powell, Sparta. New Perspectives, Londres, 1999, p. 189-210.

44. Pausanias, III, 12,5 et $14,2$.

45. IG V1210-212; cf. Hésychios, Tau 33 Schmidt.

46. D33 et C30.

47. Pausanias, III, 11, 2 (rôle des Bidiaioi); 14, 8-10 et 20, 2 avec Lucien, Anacharsis, 38 (description du Platanistas, forme des combats et sacrifices préliminaires); $c f$. Plutarque, Moralia (Questions romaines), 290D (sacrifice des chiots) et Hérodote, VI, 61 (localisation du Phoibaion). 
Un combat entre deux sangliers était également organisé, qui présageait de l'issue du combat du lendemain. Avant le combat, les éphèbes sacrifiaient encore à Achille, dans un sanctuaire situé à la limite nord de la ville qui, le reste du temps, demeurait fermé ${ }^{48}$. Pausanias ne précise pas si ce sacrifice était collectif ou individuel, ni s'il avait lieu avant ou après le rite effectué au Phoibaion. Le sanctuaire d'Achille se trouvait à la limite du centre urbain, sans qu'il soit possible de préciser s'il était en deçà ou au-delà des murs, en un point où il fallait sans doute passer pour se rendre au Phoibaion.

La fête des Hyacinthies donnait lieu, quant à elle, à une procession qui reliait la ville au sanctuaire d'Apollon à Amyclées. Au second jour de la fête, qui en comptait trois, des jeunes filles défilaient dans des kannathra, sortes de chars en osier, dont Plutarque nous apprend qu'ils avaient la forme d'animaux fabuleux ${ }^{49}$. C'est par Xénophon que l'on sait que cette procession en kannathra avait pour but Amyclées $^{50}$. Il y avait à Sparte une « voie Hyakinthis», qui devait être la voie processionelle vers l'Amyclaion empruntée lors de la procession ${ }^{51}$. Plusieurs indices laissent supposer que cette procession était encore - ou à nouveau - organisée à l'époque impériale. Il est possible que Plutarque ait vu les kannathra dont il donne la description, à moins qu'il n'ait repris celle-ci à un témoignage antérieur. Plus significative, sans doute, est la réapparition de la statue d'Apollon Amyclaios sur des monnaies de Sparte aux types de Commode et de Galien ${ }^{52}$. Pour les auteurs des II $^{\mathrm{e}}$ et III $^{\mathrm{e}}$ siècles, les Hyacinthies sont en tout cas une fête emblématique de la cité ${ }^{53}$, mais cela illustre peut-être davantage leur connaissance des traditions religieuses de Sparte que des pratiques de leur temps. On conserve aussi le nom de deux femmes : Memmia Xenocratia et Pompeia

48. Pausanias, III, 20, 8 .

49. Plutarque, Vie d'Agésilas, 19,5 .

50. Xénophon, Agésilas, VIII, 7. Voir encore Ovide, Métamorphoses, X, 219, avec Martin Persson Nilsson, Griechische Feste von religiöser Bedeutung, Leipzig, 1906, p. 136.

51. Athénée, IV, 173f-174a (= Demetrios de Skepsis, fr. 10 Gaede : II siècle avant J.-C.).

52. Suzanne Grunauer-von Hœrschelmann, Die Münzprägung der Lakedaimonier, Berlin, 1978 [Deutsches archäologisches Institut. Antike Münzen und geschnittene Steine, Bd. VII], p. 99.

53. Outre Athénée, IV, 139d-f (= Polykrates, FGrH 588 F 1; cf. Schmidt, p. 44) et IV, 138e (= Polemon, fr. 86 Preller) qui décrit le déroulement de la fête, voir Philostrate, Vie d'Apollonios de Tyane, VI, 20, 1. 
Polla, qui furent, à la fin du $\mathrm{II}^{\mathrm{e}}$ et au début du $\mathrm{III}^{\mathrm{e}}$ siècle, archèis et théores à vie du très auguste concours des Hyacinthies ${ }^{54}$. D'une génération plus jeune que Pompeia Polla, Memmia Xenocratia a selon toute vraisemblance succédé à Pompeia Polla dans ses charges, que l'une et l'autre ont exercées alors qu'elles étaient assez âgées ${ }^{55}$. Leur tâche impliquait peut-être la prise en charge de la confection de la tunique d'Apollon Amyclaios qui, selon Pausanias, était tissée par les femmes de Sparte dans un local dédié à cette tâche ${ }^{56}$. Peut-être la tunique était-elle acheminée à l'occasion de la fête du dieu, au cours de la procession. Une autre procession encore est connue de Pausanias :

«il se trouve un sanctuaire de Déméter dont le surnom est Éleusinia («Éleusinienne »). Les Lacédémoniens affirment que c'est là qu'Héraclès, qui s'efforçait de soigner sa blessure, a été caché par Asclépios. Et à l'intérieur il y a une statue ancienne d'Orphée, œuvre de Pelasges, à ce que l'on affirme. Et je sais que l'on y fait ceci encore. Près de la mer se trouvait la bourgade d'Hélos ( Le Marécage »), dont Homère a fait mention dans le catalogue des Lacédémoniens [...]. De cette Hélos, on emmène certains jours déterminés une statue ancienne de Coré fille de Déméter vers l'Éleusinion $»^{57}$.

La procession aurait donc relié un des sanctuaires périphériques de Sparte à une ancienne cité, que Pausanias signale ailleurs comme disparue et qui était située dans la plaine côtière de l'Eurotas, à environ $35 \mathrm{~km}$ de l'Éleusinion. Hélos, ou plutôt le site qui lui était identifié, aurait donc abrité un sanctuaire de Coré, comme Bryséai, autre cité homérique, accueillait celui de Dionysos. Cela n'a rien d'impossible, mais d'autres éléments suggèrent une interprétation différente. La localisation «dans l'Hélos » est en effet associée à plusieurs cultes mentionnés dans les inscriptions de Sparte. Vu leur nombre - au moins trois, et sans doute plus de sept : les textes sont ambigus -, il parait plus vraisemblable que l'Hélos en question soit un quartier de Sparte plutôt que l'antique cité côtière ${ }^{58}$. Parmi les

54. IG V1 586 et 587.

55. Du moins, elles étaient déjà âgées lorsqu'elles ont été honorées d'une statue.

56. III, $16,2$.

57. III, 20, 5-7.

58. Voir Patrick Marchetti, Kostas Kolokotsas, Le nymphée de l'agora d'Argos: fouille, étude architecturale et historique, Athènes, 1995 [Études Péloponnésiennes, 11], p. 211-216. C'était déjà l'avis de August Boeckh, Corpus Inscriptionum Graecarum, Berlin, 1828, n ${ }^{\circ}$ 1444, suivi par Christos Tsountas, 
sanctuaires qui se trouvaient certainement « dans l'Hélos » se trouvait celui de Coré $^{59}$. La déesse apparaît dans une série de cultes dont la prêtrise était détenue à la fin du II $^{\mathrm{e}}$ et au début du $\mathrm{III}^{\mathrm{e}}$ siècle après J.-C. par la famille des Tiberii Claudii. Parmi ces dieux se trouve Carneios Oiketas dont le sanctuaire, nous dit Pausanias, se trouvait près de l'agora de Sparte, dans la même zone qu'un temple de Coré Sôteira. Celle-ci doit être la Coré « dans l'Hélos » d'où était acheminée la statue jusqu'à l'Éleusinion. Le lien de l'Éleusinion avec ce culte urbain est renforcé par la présence d'Orphée, qui avait une statue dans le premier, alors que, selon Pausanias, il avait bâti à Sparte le temple de Coré Sôteira ${ }^{60}$.

Les traditions religieuses et le rituel associent donc étroitement le sanctuaire de Déméter Éleusinia et celui de Coré à l'époque impériale. Or ce lien était encore renforcé, du moins au début du $\mathrm{III}^{\mathrm{e}} \mathrm{s}$., au niveau du personnel desservant ces sanctuaires. Ainsi Claudia Damosthéneia, qui détenait la prêtrise gentilice de la Coré de l'Hélos, était également thoinarmostria, maîtresse des banquets, à l'Éleusinion, dont le culte était à l'époque sous la responsabilité de sa cousine, la prêtresse Claudia Agéta.

\section{Les femmes de Sparte à l'Éleusinion}

Le sanctuaire de l'Éleusinion, qui a été en partie fouillé en avril 1910 et en novembre $1949^{61}$, reste relativement mal connu. Le site a livré du matériel des époques géométrique tardive, archaïque, classique, hellénistique et romaine, avec une nette prédominance pour ces deux dernières. La présence de tuiles et de quelques éléments d'architecture atteste l'existence d'un ou plusieurs bâtiments que les fouilles n'ont cependant pas permis de localiser. On

Archaiologiki Ephimeris, 1892, c. 26 et Marcus N. Tod, in : Marcus N. Tod, Allan J.B. Wace, A Catalogue of the Sparta Museum, Oxford, 1906, p. $81 \mathrm{n}^{\circ}$ 691. Anette Hupfloher, Kulte im kaiserzeitlichen Sparta. Eine Rekonstruktion anhand der Priesterämter, Berlin, 2000, p. 82-83, défend la position inverse.

59. IG V1 497 1. 16-17; $5891.10-11 ; 6081$ 1. 5-6.

60. III, 13, $2 ; c f$. III, 14, 5.

61. $C f$. Richard M. Dawkins, «Laconia. I. Excavations at Sparta, 1910, 3. The Eleusinion at Kalyvia tes Sokhas », ABSA 16 (1909-1910), p. 12-14; John M. Cook, «Kalyvia Sokhas », ABSA 45 (1950), p. 261-281, pl. 26-27; Conrad M. Stibbe, « Das Eleusinion am Fuße des Taygetos in Lakonien », Bulletin Antieke Beschaving 68 (1993), p. 71-105. 
reconnaît sur plusieurs tuiles le nom de Déméter ${ }^{62}$, mais celle-ci est associée à Coré dans les dédicaces ${ }^{63}$ et dans la titulature des desservantes du culte ${ }^{64}$.

Plusieurs inscriptions provenant du sanctuaire ont été conservées, notamment une vingtaine de bases qui nous permettent de connaître quinze femmes, exclusivement, ayant eu l'honneur de voir leur statue érigée dans le sanctuaire. Il est fait mention, pour certaines d'entre elles, de la charge qu'elles ont exercée, le plus souvent comme maîtresses des banquets ou comme pôlos - une fonction subalterne de nature indéterminée. Comme il se doit, la piété de ces femmes est régulièrement soulignée. Seule une prêtresse, Iulia Apatarion, apparaît dans ces textes, non comme destinataire d'une de ces statues mais en tant que grand-mère de l'une d'elles.

De la même époque que cette série de bases datent trois reliefs votifs d'un type très particulier, qui proviennent également du sanctuaire. Deux ont été trouvés en octobre 1803 dans une chapelle du village de Sklavochori par George Hamilton Gordon, quatrième comte d'Aberdeen, et sont aujourd'hui conservés au British Museum. Ils avaient déjà été vus par l'abbé Michel Fourmont, qui en avait donné une description totalement farfelue ${ }^{65}$. Ce sont deux plaques de marbre gris, de respectivement 89 sur $89 \mathrm{~cm}$ et $107 \mathrm{sur} 69 \mathrm{~cm}$, pour une épaisseur identique de $26 \mathrm{~cm}$. Ils représentent en bas-relief une série d'objets encadrés dans une guirlande végétale. Un troisième relief comparable, découvert par l'archéologue grec Rhomaios en 1906, a été remployé comme chapiteau dans un monastère à quelques kilomètres de l'Éleusinion ${ }^{66}$. Au centre de chacun de ces reliefs est

62. R.M. Dawkins, «Laconia. I», art. cité, p. 13, fig. $1 d$ (= C.M. Stibbe, ibid., p. $104, \mathrm{n}^{\circ} 39$ et p. 96 , fig. $\left.65 ; c f . I G \mathrm{~V} 11515 \mathrm{~d}\right)$ et $f\left(=\mathrm{n}^{\circ} 38\right.$ et fig. $64 ; I G$ V1 1515e). Sur les tuiles $b\left(=\mathrm{n}^{\circ} 47\right.$ et fig. 63$)$ et $g(I G \mathrm{~V} 11515 \mathrm{~h})$, où l'on reconnaît les lettres majuscules delta, alpha et mu, on ne peut exclure qu'il s'agisse du début du mot damosios ( $c f . I G$ V1 850 et suiv.).

63. Marcus N. Tod, « Notes on Some Inscriptions from Kalyvia Sokhas », ABSA 47 (1952), p. 122 (SEG XI 885a); cf. l'inscription éditée par J. M. Cook, «Kalyvia Sokhas », art. cité, p. 278-280 (d'où SEG XI 812a) et revue par E. Kourinou-Pikoula, «Epigraphes apo ti Sparti », HOROS 4 (1986), p. 68-69, $\mathrm{n}^{\circ} 6$ et pl. 9.4 (d'où $S E G$ XXXVI 353).

64. $I G$ V1 583 11. 8-9; $6061.7 ; 607$ 11. 27-28.

65. Voir la lettre d'Aberdeen publiée par Robert Walpole, Travels in various Countries of the East, Londres, 1820, p. 489-503 avec Richard Stoneman, "The Abbé Fourmont and Greek archaeology », Boreas 8 (1985), p. 190-198.

66. Sans doute s'agit-il du relief que A. Hupfloher déclare avoir vu dans les réserves du musée de Sparte : A. Hupfloher, Kulte im kaiserzeitlichen Sparta, 
représentée une coupe, sur le fond de laquelle était gravée une inscription. Celle du troisième relief a été rendue illisible par la gravure de symboles chrétiens, mais on peut lire sur les exemplaires du British Museum le nom et la fonction des dédicantes. L'une était assistante, l'autre prêtresse. Les objets représentés sur ces reliefs étaient peutêtre utilisés par elles dans l'exercice de leur sacerdoce au service de la déesse. Ils appartiennent en tout cas tous au monde féminin, et spécialement au domaine de la parure (miroirs, boîtes à fard, mortier, bouteilles à parfums, etc.), mais pas exclusivement. Susan Walker, qui a étudié récemment les exemplaires du British Museum, suppose qu'il s'agissait des objets utilisés par les prêtresses pour se parer en vue de la fête de la déesse Déméter ${ }^{67}$. Les reliefs représenteraient un substitut plus durable à l'offrande des objets eux-mêmes.

Certaines de ces femmes ne sont pas autrement connues, mais il est possible de retracer la généalogie d'une dizaine d'entre elles de manière plus ou moins assurée ${ }^{68}$. Les femmes qu'il est possible d'identifier se répartissent sur six générations et ont été actives dans le sanctuaire du début du $\mathrm{II}^{\mathrm{e}}$ siècle au milieu du $\mathrm{III}^{\mathrm{e}}$ siècle environ. Elles appartiennent pour l'essentiel à deux grandes familles de Sparte : les Publii Memmii, de la branche des Pratolai, et les Tiberii Claudii. On identifie également deux femmes de la famille des Iulii, alliées à ces familles. L'une d'elles, la prêtresse Iulia Apatarion, doit être née dans la seconde moitié du $\mathrm{I}^{\mathrm{er}}$ siècle après J.-C., mais elle était encore en charge, semble-t-il, dans le second quart du II $^{\mathrm{e}}$ siècle, lorsque sa petite fille Claudia Nicion a été honorée dans le sanctuaire. La seconde prêtresse connue, Claudia Agéta, pourrait être une arrière arrière petite-fille de Iulia Apatarion. Ce fait est important, car l'inscription qui la mentionne précise que la prêtrise appartenait à sa famille (apo genous), et Claudia Agéta pourrait donc l'avoir tenue d'elle. La charge de thoinarmostria, la plus attestée dans les textes,

op. cit., p. 53 n. 62. Pas plus que Susan Walker (voir infra note 67), elle ne semble avoir eu connaissance de l'article de Rhomaios.

67. Susan Walker, "Two Spartan women and the Eleusinion", in : Susan Walker, Averil Cameron (éd.), The Greek Renaissance in the Roman Empire, Londres, 1989, p. 130-141

68. L'identité des femmes honorées à l'Éleusinion dont il est possible de retracer la généalogie est répertoriée dans le tableau ci-joint [fig. 1]; leur nom apparaît en caractères gras. Pour les références, voir Athanasios D. Rizakis, S. Zoumbaki, C. Lepenioti, Roman Peloponnese, II. Roman Personal Names in their Social Context (Laconia and Messenia), Athènes, 2004 [Meletêmata, 36]. 
était de nature liturgique et impliquait certainement des dépenses importantes. Elle a été exercée par Memmia Xénocratia, Claudia Longina et Claudia Damosthéneia - parentes plus ou moins proches des prêtresses Iulia Apatarion et Claudia Agéta -, mais également par d'autres femmes pour lesquelles aucun lien avec les deux grandes familles les mieux représentées dans le sanctuaire n'est attesté.

Selon Susan E. Alcock, les élites auraient joué un rôle moteur pour maintenir vivante l'activité cultuelle dans le territoire des cités. Elles se seraient investies à la fois en raison de l'importance des sanctuaires comme lieu de rencontre pour entretenir et développer les réseaux personnels entre familles, en raison de l'apport économique non négligeable que constituait l'organisation de fêtes et de concours, et en raison d'attaches personnelles avec les territoires où se seraient concentrés leurs intérêts économiques. Autant de motifs qui, de manière générale, mêlent le souci de se dévouer à la cité et celui de renforcer leur position sociale. Est-ce cela que nous observons à l'Éleusinion? S'il faut souligner l'omniprésence des représentants des familles des Memmii et des Claudii, ce n'est pourtant qu'une tendance. Seule la transmission de la prêtrise au sein d'une même famille élargie pourrait être vue comme la trace d'une mainmise sur le sanctuaire, mais ce n'est là ni un phénomène isolé en Grèce, ni une tendance nouvelle à Sparte ${ }^{69}$. D'autres femmes ont exercé des charges dans le sanctuaire, d'autres femmes y ont été honorées et, dans l'ensemble, les liturgies assumées dans le sanctuaire par les épouses des plus riches citoyens de Sparte ou les honneurs qu'elles y ont reçu correspondent à ce que l'on connaît pour leurs époux dans leur propre domaine d'activité. Pour établir la prééminence de certaines familles dans le sanctuaire, il faudrait trouver trace, nous semble-t-il, de bienfaits autrement durables et importants. En revanche, les inscriptions de l'Éleusinion révèlent, une fois encore, l'endogamie prononcée des familles les plus riches de Sparte $^{70}$.

69. Sur ce type de prêtrises - qu'il faut peut-être qualifier de gentilices plutôt que de prêtrises héréditaires dans la mesure où leur transmission pouvait répondre à des procédures plus complexes qu'une simple transmission de père/mère à fils/ fille -, voir Jean-Sébastien Balzat, « Prosopographie des prêtres et prêtresses des Dioscures de la Sparte d'époque impériale », in: Athanasios D. Rizakis (éd.), Roman Peloponnese, III, Athènes, 2010 [Mélétêmata 63], p. 341-355.

70. Phénomène bien mis en valeur par A.J.S. Spawforth. Voir en dernier lieu P. Cartledge \& A.J.S. Spawforth, Hellenistic and Roman Sparta, p. 160-165. 
L'omniprésence des femmes à l'Éleusinion s'explique par la nature du culte desservi. Mais la multiplication des statues honorifiques sur une assez courte période constitue une évolution très particulière des marques de dévotion, d'autant qu'elle se double de la célébration de la générosité des officiantes pour la cité et le sanctuaire. La comparaison avec d'autres sanctuaires est assez éclairante. Les deux bases, déjà évoquées, de statues provenant de l'Amyclaion sont tout à fait comparables à celles de l'Éleusinion, et concernent d'ailleurs les mêmes femmes. Peut-être une exploration plus systématique du sanctuaire apportera-t-elle d'autres exemples. Au Ménélaion en revanche, rien de tel. Même si le sanctuaire a continué d'être visité - et même s'il a peut-être accueilli une activité cultuelle qui n'aurait pas laissé de traces archéologiques -, il n'a pas connu le développement monumental de l'Éleusinion où fleurissent statues et reliefs votifs.

Dans la mise en scène de soi et de sa propre générosité, qui caractérise les élites de la société grecque d'époque impériale, il semble que le culte de l'Éleusinion ait offert un champ d'action tout particulier. Peut-être parce qu'il permettait, précisément, d'étendre à l'activité des femmes l'autocélébration qui saturait déjà l'espace public dans la sphère masculine des fonctions politiques. Dans la province d'Achaïe, le domaine religieux est le seul où les femmes pouvaient trouver l'occasion d'agir ${ }^{71}$. À ce titre, il est révélateur que nombre de femmes qui nous sont connues à l'Éleusinion étaient émancipées et avaient donc un pouvoir d'initiative qui trouvait ainsi à s'exprimer. Plus qu'un hypothétique lien avec les intérêts économiques détenus éventuellement par les grandes familles de Sparte dans la campagne environnante - explication qui vaudrait pour d'autres sanctuaires -, et plus que le prestige intrinsèque de celui-ci - que l'on ne peut nier, mais qu'il est difficile d'évaluer -, ce serait donc selon nous des conditions sociales particulières qui expliqueraient la monumentalisation des honneurs rendus aux femmes de Sparte et à leur piété à l'Éleusinion.

Pour autant, la nouveauté n'est peut-être qu'apparente, et ce, à double titre. D'une part, les inscriptions honorifiques ou les reliefs ont pu se substituer à d'autres manifestations destinées à honorer les

71. Riet Van Bremen, The Limits of Participation. Women and civic life in the Greek East in the Hellenistic and Roman periods, Amsterdam, 1996, p. 82. 
déesses aussi bien que la piété des femmes à leur endroit. On peut songer notamment aux dédicaces monumentales qui étaient venues orner le sanctuaire à l'époque hellénistique ${ }^{72}$. D'autre part, nous ignorons tout des pratiques cultuelles qu'accueillait le sanctuaire, des offrandes qu'on y déposait, des publics qui le fréquentaient. La visibilité des statues érigées dans le sanctuaire, qui a bien sûr en tant que telle son importance, ne doit pas faire oublier tout une part d'éphémère qui, par sa nature même, nous demeure quasiment inaccessible.

Institut für Alte Geschichte und Altertumskunde, Papyrologie und Epigraphik Universität Wien

Dr.-Karl-Lueger-Ring, 1 1010 Wien

olivier.gengler@univie.ac.at

72. Voir J.M. Cook, « Kalyvia Sokhas », art. cité, p. 263-265 et 275-276. 
CATALOGUE DES LIEUX DE CULTE DU TERRITOIRE DE LA CITÉ DE SPARTE À L'ÉPOQUE IMPÉRIALE ${ }^{73}$

\section{Zone A : Route menant à Sparte depuis la frontière avec Argos et Tégée}

A1 Paus., 10, 6: sanctuaire de Zeus Scotitas, à l'écart de la route.

A2 Paus., 10, 6 : statue d'Héraclès, à l'écart de la route. La statue est associée à un trophée qu'Héraclès aurait érigé après avoir tué Hippocoon et ses enfants.

A3 Paus., 10, 7; + Wide, p. 102-103: sanctuaire d'Artémis Caryatis, à Caryai. Le lieu est consacré à Artémis et aux Nymphes; il y a une statue d'Artémis en plein air; des jeunes filles de Lacédémone y dansent à la mode du pays.

A4 Hésychios, Sigma 370 et 371 Hansen: Artémis Sel(l)asia, à Sel(1)asia. D'après Pausanias, Sellasia était en ruine à son époque, suite à sa destruction complète en 222 avant J.-C., mais il est possible qu'un culte y ait perduré.

A5 Paus., 10, 8; Hésychios, Thêta 647 Latte; $c f$. Xénophon, Helléniques, VI, 5, 27 : statue (sanctuaire, Hés.; temenos, Xén.) d'Apollon Pythéen (Thornakios, Hés.), à Thornax.

\section{Zone B : Au sud de la ville}

B6* Chrysanthos Christou, Ergon, 1962, p. 135-137 (cf. Praktika, 1962, p. 115-116; AD 18 (1963), p. 86) : sanctuaire d'époque archaïque, à Kalogônia. Petit bâtiment rectangulaire de $12,70 \mathrm{~m}$ sur $8,90 \mathrm{~m}$, peut-être rénové à l'époque romaine.

B7 Paus., 18, 6: sanctuaire des Charites Phaénna et Cléta, près de la rivière Tiasa sur la route menant de Sparte à Amyclées. Fondé par Lacédémon, qui a aussi établi le nom des Charites.

B8 Athénée, IV, 16, 139b citant Polémon (= Karl Müller, Fragmenta Historicorum Graecorum, t. III, Paris, 1883, fr. 86,

73. Sauf mention contraire, les références à Pausanias (Paus.) concernent le livre III ; l'astérisque * signale les sanctuaires localisés (voir la carte ci-jointe). La liste des abréviations utilisées ici se trouve à la suite du catalogue. 
p. 142-143); + Wide, p. 101 : sanctuaire d'Artémis Corythalia, près de la rivière Tiasa et du sanctuaire de Cléta $\mathrm{B} 7$.

B9* Paus., 18, 7-19, 5 ; + Wide, p 67-69 et p. 285-289 : sanctuaire d'Apollon, à Amyclées. Le héros Hyakinthos y recevait un culte à côté d'Apollon. Fête des Hyacinthies avec une procession. Localisé sur la colline d'Aghia Kyriaki, en partie fouillé : Christos Tsountas, Archaiologiki Ephimeris, 1892, c. 1-26; Klaus Fiechter, Jahrbuch des Deutschen Archäologischen Instituts 33 (1918), p. 107-245; Ernst Buschor, Wilhelm von Massow, Mitteilungen des Deutschen Archäologischen Instituts. Athenische Abteilung 52 (1927), p. 1-85; cf. Peter G. Calligas, «From the Amyklaion», in : Jan M. Sanders (éd.), Philolakôn. Lakonian Studies in Honour of Hector Catling, Londres, 1992, p. 31-48; LS, II, p. 290, GG88. Nouvelles fouilles entreprises par Angelos Delivorrias en 2004 (AR 54 (2007-2008), p. 36). Le site de l'Amyklaion, accueillait déjà un lieu de culte à l'Helladique Récent IIIB et C (vers 1350-1050 avant J.-C.); après une interruption d'un siècle environ, l'occupation du site est à nouveau documentée à partir de 950 avant J.-C. env. et jusqu'à la fin du monde antique.

B10* Paus., 19, 6; + Wide, p. 333-334 : sanctuaire et tombeaux d'Alexandra et d'Agamemon, à Amyclées. Localisés près de l'église d'Aghia Paraskevi, à $700 \mathrm{~m}$ au sud du sanctuaire d'Apollon B9, en partie fouillé : Ergon, 1956, p. 100-104 et fig. 103-108; Praktika, 1956, p. 211-212; Praktika, 1960, p. 228-231 et pl. 170-171; Praktika, 1961, p. 177-178; LS, II, p. 290, GG92; $A D, 53$, B.1, 1998, p. 173 ; dépôt votif constitué de plusieurs milliers d'objets s'échelonnant du viI ${ }^{\mathrm{e}}$ siècle avant J.-C. à l'époque hellénistique. Identification des destinataires du culte assurée par la découverte de fragments de vases inscrits.

B11 Paus., 19, 6 : Dionysos Psilax, à Amyclées.

B12 Paus., 20, 2 : sanctuaire de Poseidon Gaiaochos, non loin du Phoibaion. Une course d'attelage y est attestée à l'époque classique $(I G \mathrm{~V} 1,2131.9)$.

B13 Paus., 20, 3 : hérôon de Lacédémon, à Alésiai. Lieu où Mylès a broyé pour la première fois le grain. L'hérôon pourrait être voisin du sanctuaire des Charites B7, fondé par Lacédémon et situé au sud de la ville. 
B14* Paus., 20, 3; cf. Stephane de Byzance, s.v. Messapeai: temenos de Zeus Messapeus, dans la plaine. Peut-être localisé à Antochori: Ch. Christou, Ergon, 1962, p. 134-5 (cf. Praktika, 1962, p. 113-115; AD 17 (1961-1962), B, p. 84-85 et $A D 18$ (1963), p. 86; $L S$, II, p. 293, GG108); Ioannis G. Taïphakos, «Lakônikai epigraphai anekdotoi », Peloponnisiaka 12 (1976-1977), p. 214-223, aux p. 219-222, $\mathrm{n}^{\circ} 3$ (SEG XXVI 460). Le matériel s'échelonne de l'époque mycénienne à l'époque byzantine.

B15 Paus., 20, 3 : temple de Dionysos, à l'emplacement de l'antique Bryséai. La statue est en plein air; seules les femmes ont accès au temple et font les sacrifices, en secret.

B16 Paus., 20, 4: sommet du Taygète, le Taleton, consacré à Hélios. On y sacrifie des chevaux. Un culte de Zeus Taletitas est connu par le calendrier cultuel $I G \mathrm{~V} 1363$ (=LSG 62).

B17* Paus., 20, 5-7 : sanctuaire de Déméter Éleusinia, au pied du Taygète. Héraclès y aurait été caché par Asclépios pour y soigner sa blessure; il s'y trouve un xoanon d'Orphée; on y mène un xoanon de Coré en procession depuis l'Hélos. Localisé, en partie fouillé (voir ci-dessus).

B18 Paus., 20, 7; + Wide, p. 103 : sanctuaire d'Artémis Déréatis, au Déreion. La statue est en plein air, près d'une source.

B19 Paus., 21, 4 : statue de Zeus Crokéatas, à Crokéai, sur la route vers Gythion.

B20 Paus., 21, 4: statues des Dioscures, près de la carrière de Crokéai, sur la route vers Gythion.

B21 Paus., 21, 5 : temple de Poseidon, à Aigiai, sur la route vers Gythion. Le temple se trouve sur un étang sacré où il est interdit de pêcher; il y a aussi une statue du dieu.

B22* Zisis Bonias, Ena agrotiko iero stis Aigies Lakônias, Athènes, 1998: sanctuaire d'Artémis et du héros Timagenès (?). Petit sanctuaire semi rupestre en périphérie du site d'Aigiai ayant livré du matériel daté de l'époque archaïque jusqu'au $\mathrm{IV}^{\mathrm{e}}$ siècle après J.-C.

B23 Paus., 24, 8 : sanctuaire d'Asclépios et d'Artémis Daphnaia, au lieu-dit Hypsoi, à 30 stades de Las.

B24 IG V1602: sanctuaire d'Artémis Patriotis, à Pleiai (audessus d'Acriai : Tite Live, XXXV, 27, 2). 


\section{Zone C : Thérapné et la zone à l'est de la ville}

C25* Hector W. Catling, «A Sanctuary of Zeus Messapeus : Excavations at Aphyssou, Tsakona, $1989 »$, ABSA 85 (1990), p. 15-35 et pl. 3-6 (cf. Id., « Zeus Messapeus near Sparta : An interim Report », Lakônikai Spoudai 10 (1990), p. 276-295; $L S$, II, p. 390, N415); $A R 46$ (1999-2000), p. 43 : sanctuaire de Zeus Messapeus, au nord-est de Sparte, au lieu-dit Tsakona. Destinataire du culte identifié par des inscriptions (dédicace : SEG XXXIX 376; quatre ou cinq tuiles estampées : SEG XL 358). Pas de trace d'occupation après le début de l'époque hellénistique et jusqu'aux $\mathrm{III}^{\mathrm{e}}-\mathrm{IV}^{\mathrm{e}}$ siècle après J.-C., lorsque le culte semble reprendre sous une forme nouvelle.

C26 Paus., 19, 7: temple d'Asclépios Cotyleus, sur la route vers Thérapné. Construit par Héraclès après avoir été guéri d'une blessure à la hanche reçue lors d'un combat avec Hippocoon.

C27 Paus., 19, 7-8 : sanctuaire d'Arès Théritas, à gauche de la route vers Thérapné. La statue a été apportée par les Dioscures de Colchide.

C28 Paus., 20, 1 : sanctuaire de Pollux, à droite de la route vers Thérapné. Le sanctuaire est près d'une source.

C29* Paus., 19, 9; + Wide, p. 340-342 : temple de Ménélas, à Thérapné. Hélène et Ménélas y seraient enterrés. Localisé, fouillé : $L S$, II, p. 401-406, Q360. Plusieurs inscriptions attestent qu'Hélène était honorée dans le sanctuaire dès le $\mathrm{VII}^{\mathrm{e}}$ siècle avant J.-C.; la plus ancienne (SEG XXVI 457) mentionne Hélène comme épouse de Ménélas (vers 675-650 avant J.-C.). Deux inscriptions confirment également la présence de Ménélas à l'époque archaïque. Le site n'a livré aucun vestige postérieur à l'époque hellénistique et semble dès lors déserté, mais il devait être encore fréquenté à l'époque de Paus., comme l'indiquent certaines trouvailles isolées ( $c f$. Hector W. Catling, «Excavations at the Menelaion, Sparta, 1973-1976», AR, 23 (1976-1977), p. 41 et fig. 52).

C30 Paus., 20, 2; + Wide, p. 304-305 : temple des Dioscures, au Phoibaion, non loin de Thérapné. Les jeunes gens y sacrifient à Enyalios. 


\section{Zone D : Route depuis Sparte vers l'Arcadie}

D31* Guy Dickins, "A sanctuary on the Megalopolis road», ABSA 13 (1906-1907), p. 169-173; Conrad M. Stibbe, « The "Achilleion" near Sparta. Some unknown finds », in : Robin Hägg (éd.), Peloponnesian Sanctuaries and Cults, Stockholm, 2002 (Skrifter utgivna av svenska institutet $\mathrm{i}$ Athen, $4^{\circ}$ XLVIII), p. 207-219: sanctuaire anonyme, attribué par l'inventeur à Achille ( $c f$. D33). Le matériel découvert, datant surtout de l'époque archaïque, ne permet aucune identification. L'absence de trace de culte à l'époque impérial n'est pas totalement assurée étant donné les conditions de fouille et de publication.

D32 Paus., 20, 8 : statue d'Athéna Pareia, à moins de 30 stades de Sparte. La statue est en plein air.

D33 Paus., 20, 8 : sanctuaire d'Achille, à moins de 30 stades de Sparte. Le temple est fermé; les jeunes gens sacrifient à Achille avant les combats au Platanistas; il a été fondé par Prax, un descendant de Néoptolème.

D34 Paus., 20, $9:$ statues des planètes, à moins de 30 stades de Sparte.

D35 Paus., 20, 9 : temenos de Cranios Stemmatios, à moins de 30 stades de Sparte.

D36 Paus., 20, 9: sanctuaire d'Artémis Mysienne, à moins de 30 stades de Sparte.

D37 Paus., 20, 10-11 : statue d'Aidôs, à 30 stades de Sparte. Consacrée par Pénélope.

D38 Paus., 21, 2 : sanctuaire d'Asclépios, à Pellana.

\section{Localisation inconnue}

X39 IG V1 559 : sanctuaire de Sôstratia, à Egeila (ou Egeiloi). Il y avait un sanctuaire de Déméter à Aigila d'après l'inscription concernant le culte d'Andania ( $I G$ V1 $1390=L S G 65$ A 31). Aigila ou Aigilia est aussi le nom de l'île appelée aujourd'hui Anticythère; on y vouait un culte à Apollon Aigileus (IG V1 948). 


\section{LISTE DES ABRÉVIATIONS}

ABSA = Annual of the British School at Athens.

$A D=$ Archaiologikon Deltion .

$A R=$ Archaeological Reports.

IG V1 = Inscriptiones Graecae, t. V, fasc 1 : Laconia et Messenia, éd. par Walter Kolbe, Berlin, 1913.

$L S=$ William Cavanagh, Richard Catling, Joost Crouwel., Continuity and Change in a Greek Rural Landscape: the Laconia Survey, I: Methodology and Interpretation, Londres, 2002 [British School at Athens, Supplementary vol. 26]; II : Archaeological Data, Londres, 1996 [British School at Athens, Supplementary vol. 27].

$L S G=$ Franciszek Sokolowski, Lois sacrées des cités grecques, Paris, 1969.

SEG $=$ Supplementum Epigraphicum Graecum.

Wide = Sam Wide, Lakonische Kulte, Leipzig, 1893 [réimpression Darmstadt, 1973]. 
636 OLIVIER GENGLER

\section{ILLUSTRATIONS}

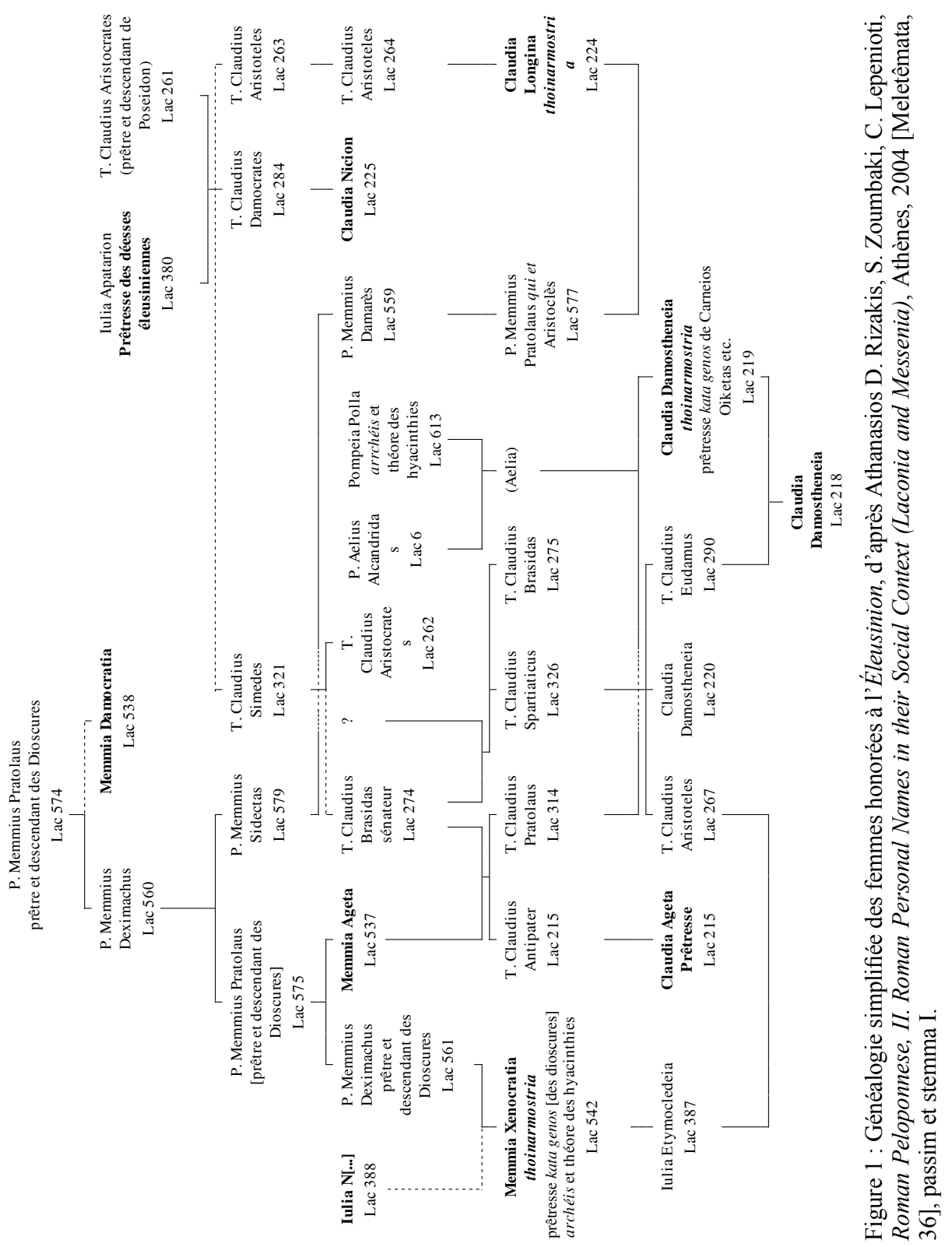




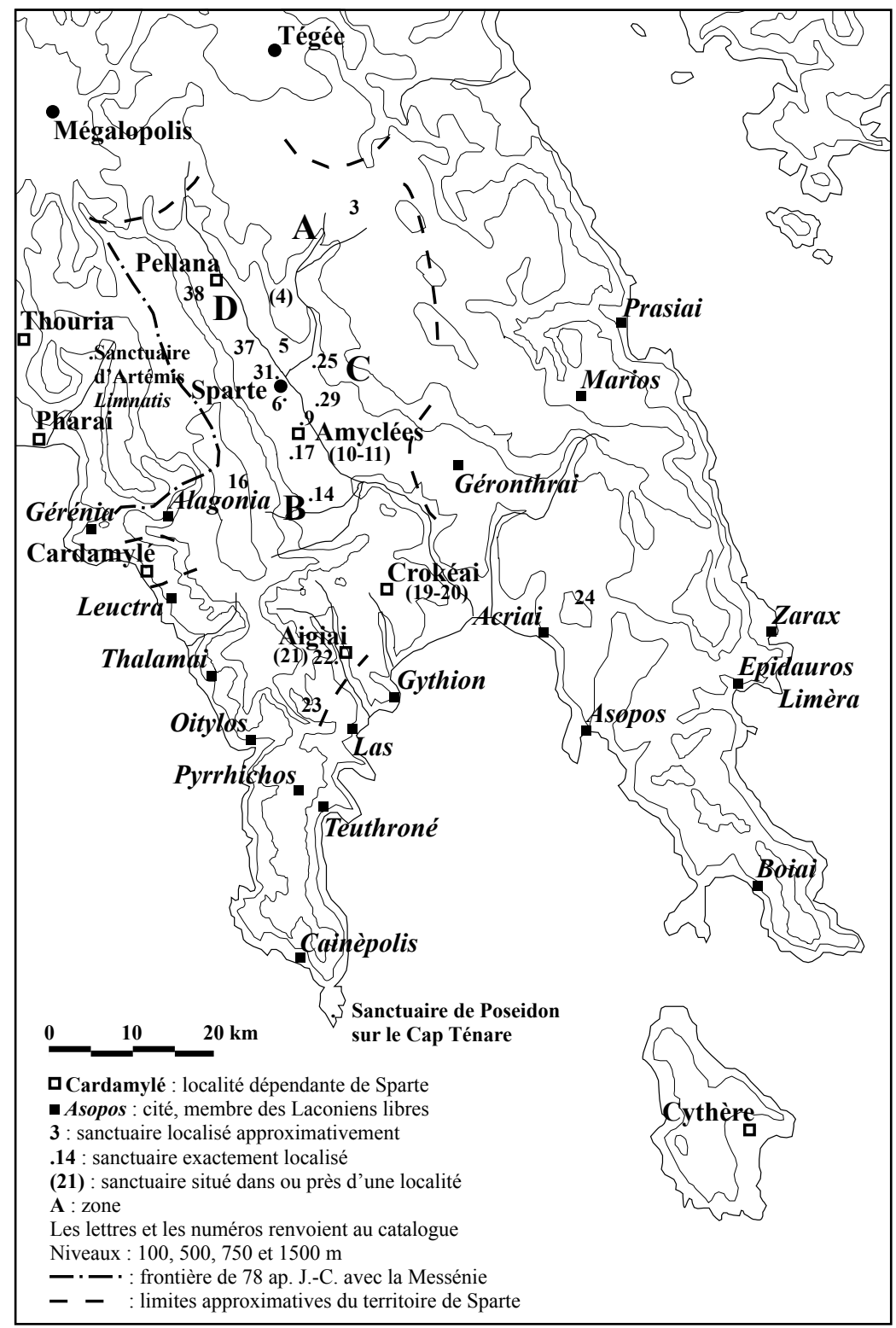

Fig. 2. Sanctuaires sur le territoire de Sparte sous le Haut-Empire (les numéros renvoient au catalogue). 